\title{
Potential regularization as the method accounting for short-range correlations in the electron liquid theory
}

\author{
M.V.Vavrukh, N.L.Tyshko \\ Ivan Franko National University of Lviv, Department of Astrophysics, \\ 8 Kyrylo i Mefodii Str., Lviv, UA-79005, Ukraine
}

Received April 1, 2004

In our previous paper [1] we proposed a Coulomb potential regularization as one of the methods for short-range correlation accounting in the electron liquid model. The present paper formulates the criterions of optimal choice of regularization as well as calculates the energetic, structural and dielectric characteristics of the model.

Key words: short-range correlation, local-field correction function, correlation energy, binary distribution function, compressibility

PACS: 05.30.Fk

\section{Introduction}

As known, the random-phase approximation (RPA) is a universal method for long-range correlation accounting in the fermi-particles systems (for example, in degenerated electron systems with Coulomb interaction). The generally accepted method for short-range correlation accounting in the modern theory of fermi-systems is based on the local-field concept. Since, the calculation of the local-field correction function (LFCF) for systems with intermediate and strong non-ideality is complicated, only approximate solutions are available [2]. LFCF was investigated in the common patterns but its microscopic theory is unfinished so far. In this situation looking for alternative methods accounting for the short-range correlation between particles remains urgent. It is very important to develop a simple and effective method of calculating the characteristics of the metallic system models.

A simple and promising approach to the evaluation of this problem can be based on the idea of correlation modelling for the degenerated fermi-systems particles with Coulomb interaction. This idea has emerged due to the model approach to the description of electron-ion interactions in metals. The modelling of interaction potential between particles is equivalent to LFCF modelling. Similarly to the electron-ion interaction theory, the effective interaction potential between electrons in an elec- 
tron liquid model (ELM) should be chosen weak at short distances and tends to the Coulomb potential $e^{2} / r$ at long distances. This approach permits to restrict ourselves to the variants of perturbation theory that were developed for a description of weak non-ideal systems in calculating the ELM characteristics in a wide range of the coupling parameter. The idea of the model effective potential of interaction between electrons can be derived from the Heisenberg indeterminacy principle. Indeterminacy of the electron position is close to the de Broglie wavelength $\lambda$. Therefore, electrons can be described as spatially distributed charged particles of a linear size about $\lambda$. The effective potential of interaction of two "smeared" electrons that defines their cross-correlation, should have the properties of quantum wave packets interaction potential

$$
V_{\mathrm{ef}}(r)=\frac{e^{2}}{r} f\left(\frac{r}{\lambda}\right)
$$

where $f(r / \lambda)$ is the dimensionless linear function at $r \ll \lambda$ and tends to unity in case of $r \gg \lambda\left(V_{\text {ef }}(r) \simeq e^{2} / \lambda\right.$ for $r \ll \lambda$ and $V_{\text {ef }}(r) \simeq e^{2} / r$ for $\left.r \gg \lambda\right)$. Asymptotic properties of a weak long-range potential $V_{\text {ef }}(r)$ follow from general physical principles. However, the selection of the function $f(r / \lambda)$ is ambiguous.

If $\rho(\mathbf{r})$ is charge distribution function of the "smeared" electron that satisfies the normalization condition

$$
e=\int \mathrm{d} \mathbf{r} \rho(\mathbf{r})
$$

then the interaction potential of two electrons can be written by the following expression:

$$
V_{\text {ef }}\left(\mathbf{r}_{1}-\mathbf{r}_{2}\right)=\iint \mathrm{d} \mathbf{r}_{1}^{\prime} \mathrm{d} \mathbf{r}_{2}^{\prime} \frac{\rho\left(\mathbf{r}_{1}^{\prime}-\mathbf{r}_{1}\right) \rho\left(\mathbf{r}_{2}^{\prime}-\mathbf{r}_{2}\right)}{\left|\mathbf{r}_{1}^{\prime}-\mathbf{r}_{2}^{\prime}\right|}
$$

Let us find explicit expressions for $\rho(\mathbf{r})$ selecting various functions $f(r / \lambda)$. Table 1 gives some variants of $\rho(\mathbf{r})$ selection in the form of function $|\mathbf{r}|$, Fourier-transform of these functions

$$
\rho(\mathbf{q})=\int \mathrm{d} \mathbf{r} \rho(\mathbf{r}) \mathrm{e}^{\mathrm{i} \mathbf{q r}},
$$

as well as a dimensionless function $f(r / \lambda)$.

In table 1 we used the following notations: $\tilde{\rho}(\mathbf{r}) \equiv e^{-1} \rho(\mathbf{r}), \tilde{\rho}(\mathbf{q}) \equiv e^{-1} \rho(\mathbf{q}), r^{*} \equiv$ $r / \lambda, q^{*} \equiv q \lambda ; K_{1}(z)$ is the modified first order Bessel function [3] with an asymptotic $z^{-1}$ at $z \ll 1$ and $\pi^{1 / 2}(2 z)^{-1 / 2} \exp (-z \lambda)$ at $z \gg 1$. The parameters $\gamma, \alpha, \beta, \delta$ are of the order of unity. The criterions for the selection of these parameters and variants can be formulated as the result of calculation of model physical characteristics.

Hereinafter we will use Fourier-transform for the potential:

$$
\begin{aligned}
& V_{\mathrm{ef}}(\mathbf{r})=\frac{1}{V} \sum_{\mathbf{q}} V_{\mathrm{ef}}(\mathbf{q}) \exp (\mathrm{iq} \mathbf{r}) \\
& V_{\mathrm{ef}}(\mathbf{q})=V_{q} \tilde{\rho}^{2}(\mathbf{q}), \quad V_{q}=\frac{4 \pi e^{2}}{q^{2}} .
\end{aligned}
$$


Table 1. Some variants of selecting the $\rho(\mathbf{r}), \rho(\mathbf{q})$ and $f\left(r^{*}\right)$

\begin{tabular}{|c|l|}
\hline Variant & $\tilde{\rho}(\mathbf{r}) ; \tilde{\rho}(\mathbf{q}) ; f\left(r^{*}\right)$ \\
\hline \multirow{4}{*}{1} & $\tilde{\rho}_{1}(\mathbf{r})=\gamma^{2} \lambda^{-3}\left[2 \pi^{2} r^{*}\right]^{-1} K_{1}\left(\gamma r^{*}\right)$ \\
& $\tilde{\rho}_{1}(\mathbf{q})=\gamma\left\{\gamma^{2}+\left(q^{*}\right)^{2}\right\}^{-1 / 2}$ \\
& $f_{1}\left(r^{*}\right)=1-\exp \left\{-\gamma r^{*}\right\}$ \\
\hline \multirow{4}{*}{2} & $\tilde{\rho}_{2}(\mathbf{r})=\alpha^{3 / 2} \lambda^{-3} \pi^{-3 / 2} \exp \left\{-\alpha\left(r^{*}\right)^{2}\right\}$ \\
& $\tilde{\rho}_{2}(\mathbf{q})=\exp \left\{-\left(q^{*}\right)^{2}(4 \alpha)^{-1}\right\}$ \\
& $f_{2}\left(r^{*}\right)=\sqrt{2 / \pi} \int_{0}^{\alpha r^{*}} \mathrm{~d} x \exp \left(-x^{2} / 2\right)$ \\
\hline \multirow{3}{*}{3} & $\tilde{\rho}_{3}(\mathbf{r})=\beta^{2} \lambda^{-3}\left\{4 \pi r^{*}\right\}^{-1} \exp \left\{-\beta r^{*}\right\}$ \\
& $\tilde{\rho}_{3}(\mathbf{q})=\beta^{2}\left\{\beta^{2}+\left(q^{*}\right)^{2}\right\}^{-1}$ \\
& $f_{3}\left(r^{*}\right)=1-\left\{1+\left(r^{*} \beta\right) / 2\right\} \exp \left(-\beta r^{*}\right)$ \\
\hline \multirow{4}{*}{4} & $\tilde{\rho}_{4}(\mathbf{r})=\delta^{3} \lambda^{-3}\{8 \pi\}^{-1} \exp \left\{-\delta r^{*}\right\}$ \\
& $\tilde{\rho}_{4}(\mathbf{q})=\delta^{4}\left\{\delta^{2}+\left(q^{*}\right)^{2}\right\}^{-2}$ \\
& $f_{4}\left(r^{*}\right)=1-\left\{1+\frac{11}{16} x+\frac{3}{16} x^{2}+\frac{1}{48} x^{3}\right\} \exp (-x)$ \\
& $\left(x=\delta r^{*}\right)$ \\
\hline
\end{tabular}

The function $\tilde{\rho}(\mathbf{r})$ or $\tilde{\rho}(q)$ can be modelled. In the latter case we can use the following expression

$$
\left.f\left(r^{*}\right)=1-\frac{1}{\pi} \int_{r^{*}}^{\infty} \mathrm{d} r^{*} \int_{-\infty}^{\infty} \mathrm{d} q^{*} \tilde{\rho}^{2}\left(q^{*}\right) \exp \mathrm{i} q^{*} r^{*}\right)
$$

\section{Description of the ground state of the electron liquid model in a random-phase approximation with effective potential}

In the case of the ground state of the model, the de Broglie wavelength of electron is defined by Fermi wave number. Therefore, we will use the potentials from table 1 at $\lambda=k_{\mathrm{F}}^{-1}$. To calculate the two-particle correlation function we restrict ourselves at the beginning by RPA, because $V_{\mathrm{ef}}(r)$ is a long-range but weak potential:

$$
\mu_{2}^{\mathrm{RPA}}(\mathbf{r})=N^{-2} \beta^{-1} \sum_{\mathbf{q} ; \nu} \mu_{2}^{\mathrm{RPA}}(x,-x) \mathrm{e}^{\mathrm{iq \mathbf {r }}}, \quad \mu_{2}^{\mathrm{RPA}}(x,-x)=\mu_{2}^{0}(x,-x)\left\{1+L_{\mathrm{ef}}(x)\right\}^{-1}
$$

$L_{\mathrm{ef}}(x)=V_{\mathrm{ef}}(q) V^{-1} \mu_{2}^{0}(x,-x)$. Here $\mu_{2}^{0}(x,-x)$ is spectral representation of twoparticle correlation function of the electron ideal system at $T=0 \mathrm{~K}, x=(\mathbf{q}, \nu)$, $\mathbf{q}$ is wave vector, $\nu$ is the Bose-Matsubara frequency and $r$ is the distance between two particles [8]. Having compared the formula (7) with the expression for $\mu_{2}(x,-x)$ in the local-field representation [4]

$$
\mu_{2}(x,-x)=\mu_{2}^{0}(x,-x)\left\{1+V^{-1} V_{q} \mu_{2}^{0}(x,-x)[1-G(x)]\right\}^{-1},
$$


we can see that $V_{\text {ef }}(q)$ is the modelling of a static variant of LFCF:

$$
G^{0}(q)=1-\tilde{\rho}^{2}(q)
$$

The average value of the system energy will be calculated using the expression

$$
E=E_{0}+\frac{1}{2 \beta V} \sum_{\mathbf{q}, \nu} V_{q} \int_{0}^{1} \mathrm{~d} \lambda \mu_{2}^{\lambda}(x,-x)
$$

where $E_{0}$ is the ideal system energy and $\mu_{2}^{\lambda}(x,-x)$ is spectral representation of two-particle correlation function of the model system with Fourier-representation $\lambda V_{\text {ef }}(q)$ of interaction potential. Thus, $V_{\text {ef }}(q)$ plays the role of self-consistent effective potential accounting for short-range correlation (local-field effects).

By extracting the ideal correlation contribution to the approximation $\mu_{2}^{\lambda}(x,-x) \rightarrow$ $\mu_{2}^{0}(x,-x)$ we can represent the total energy in the usual dimensionless form

$$
E=N R y\left\{\varepsilon_{0}\left(r_{s}\right)+\varepsilon_{\mathrm{HF}}\left(r_{s}\right)+\varepsilon_{c}\left(r_{s}\right)\right\}
$$

where $\varepsilon_{0}\left(r_{s}\right)$ is the ideal system energy at $T=0 \mathrm{~K}$ in Ry per electron, $\varepsilon_{\mathrm{HF}}\left(r_{s}\right)$ is the Hartree-Fock energy contribution and $\varepsilon_{c}\left(r_{s}\right)$ is the so-called correlation energy,

$$
\varepsilon_{0}\left(r_{s}\right)=\frac{3}{5}\left(\frac{\eta}{r_{s}}\right)^{2}, \quad \varepsilon_{\mathrm{HF}}\left(r_{s}\right)=-\frac{3}{2 \pi} \frac{\eta}{r_{s}}
$$

$\eta=(9 \pi / 4)^{1 / 3}, r_{s}$ is non-ideality parameter (Wigner-Brueckner parameter).

According to expressions (7), (9)

$$
\varepsilon_{c}\left(r_{s}\right)=\frac{3}{2 \pi}\left(\frac{\eta}{r_{s}}\right)^{2} \int_{0}^{\infty} \mathrm{d} u \int_{0}^{\infty} \mathrm{d} q q^{3}[\tilde{\rho}(q)]^{-2}\left\{\ln \left[1+L_{\mathrm{ef}}(q, u)\right]-L_{\mathrm{ef}}(q, u)\right\},
$$

where $q \equiv|\mathbf{q}| k_{\mathrm{F}}^{-1}, u \equiv \nu\left(2 \varepsilon_{f} q\right)^{-1}$. One of the criterions of selecting distributions $\rho(\mathbf{r})$ or $\rho(\mathbf{q})$ parameters can be based on the comparison of calculation results of the energy correlation term equation (13) with the previous ones from Monte-Carlo (MC) method [5]. In paper [6] there was proposed an approximative expression for numeric values of energy, obtained in [5] according to which

$$
\varepsilon_{c}\left(r_{s}\right)=-2 b_{0} \int_{a}^{\infty} \mathrm{d} x\left(b_{1}+x^{-1}\right)\left[1+b_{1} x+b_{2} x^{2}+b_{3} x^{3}\right]^{-1}
$$

at $a=r_{s}^{1 / 2}, b_{0}=0.0621814, b_{1}=9.81379, b_{2}=2.82214, b_{3}=0.69699$. However, the energy being an integral characteristic is not very sensitive to the approximations used in the calculation. Therefore, the mentioned criterion cannot be a single one and other criterions should be added to it that follow from other characteristics as functions of non-ideal parameter. 
As known, the binary distribution function $F_{2}(\mathbf{r})=1+\mu_{2}(\mathbf{r})$ in a traditional approximation RPA (on Coulomb potential) has non-physical negative values at short distances $\left(\left(r k_{\mathrm{F}} \leqslant 1\right)\right)$ for $r_{s} \geqslant 0.82[7]$. According to the definition $F_{2}(\mathbf{r})$ must be positive at any $r_{s}$ values. Also, from general physical principles there follows a monotonicity dependence $F_{2}(\mathbf{r})$ at short distances where there are no Fridel oscillations [2]. Thus, we have a second criterion for the selection of parameters

$$
F_{2}(0)=1+\mu_{2}(0) \geqslant 0 ; \quad\left|\mu_{2}\left(\mathbf{r}_{1}\right)\right|>\left|\mu_{2}\left(\mathbf{r}_{2}\right)\right| \quad \text { for } \quad r_{1}<r_{2} ; r_{1,2}^{*} \leqslant 1 .
$$

The next criterion can be obtained from a long-wave asymptote of the polarization operator in the static limit. The spectral representation of two-particle correlation function $\mu_{2}(x,-x)$ is determined by the polarization operator $M_{2}(x,-x)$ as follows (see, for example [8])

$$
\mu_{2}(x,-x)=M_{2}(x,-x)\left\{1+\frac{V_{q}}{V} M_{2}(x,-x)\right\}^{-1} .
$$

Using formula (7) from the last expression $M_{2}(x,-x)$ in RPA with an effective potential can be written as follows:

$$
M_{2}^{\mathrm{RPA}}(x,-x)=\mu_{2}^{0}(x,-x)\left\{1-\frac{V_{q}}{V} \mu_{2}^{0}(x,-x)\left[1-\tilde{\rho}^{2}(q)\right]\right\}^{-1} .
$$

Taking into account that $M_{2}(0,0)=\kappa N^{2} / V$ (where $\kappa=-V^{-1} \partial V / \partial p$ is compressibility of the system) we derive a relationship

$$
\frac{\kappa_{0}}{\kappa}=1-\left.\frac{4 r_{s}}{\pi \eta}\left\{\frac{1-\tilde{\rho}^{2}(q)}{q^{2}}\right\}\right|_{q=0},
$$

where $\kappa_{0}=3 V\left(2 \varepsilon_{\mathrm{F}} N\right)^{-1}$ is the compressibility of the ideal system. On the other hand, $\kappa$ can be found based on the thermodynamic relation, using ground state energy: as it is known $p=-\partial E / \partial V$, therefore, $\kappa^{-1}=V\left(\partial^{2} E / \partial V^{2}\right)$ or

$$
\frac{\kappa_{0}}{\kappa}=\frac{\partial^{2} E}{\partial V^{2}}\left\{\frac{\partial^{2} E_{0}}{\partial V^{2}}\right\}^{-1}
$$

Using expression (11) and by transition from variable $V$ to variable

$$
r_{s}=a_{0}^{-1}\left[\frac{3 V}{4 \pi N}\right]^{1 / 3}
$$

we will get the relationship

$$
\frac{\kappa_{0}}{\kappa}=1+\frac{r_{s}\left[\varepsilon_{\mathrm{HF}}^{\prime \prime}+\varepsilon_{c}^{\prime \prime}\right]-2\left[\varepsilon_{\mathrm{HF}}^{\prime}+\varepsilon_{c}^{\prime}\right]}{r_{s} \varepsilon_{0}^{\prime \prime}-2 \varepsilon_{0}^{\prime}}
$$

where $\varepsilon^{\prime}=\left(\mathrm{d} / \mathrm{d} r_{s}\right) \varepsilon\left(r_{s}\right), \varepsilon^{\prime \prime}=\left(\mathrm{d}^{2} / \mathrm{d} r_{s}^{2}\right) \varepsilon\left(r_{s}\right)$. According to the formulae $(14),(20)$, we obtain 


$$
\begin{aligned}
\frac{\kappa_{0}}{\kappa}= & 1-\frac{x^{2}}{\pi \eta}-\frac{b_{0} x^{4}}{6 \eta^{2}}\left[1+b_{1} x+b_{2} x^{2}+b_{3} x^{3}\right]^{-1} \times \\
& \times\left\{3+\frac{5}{2} b_{1} x+\left(1+b_{1} x\right)\left[b_{1}+2 b_{2} x+3 b_{3} x^{2}\right]\left[1+b_{1} x+b_{2} x^{2}+b_{3} x^{3}\right]^{-1}\right\},
\end{aligned}
$$

where $x \equiv r_{s}^{1 / 2}$. If the calculations of relationships (18) and (20) are correct, then the results must be convergent, or the relationships of (18) and (21) must be close. Namely, the values of non-ideality parameter must be close, that correspond to $\kappa_{0} / \kappa=0$. As it follows from the formula (21) the change of $\kappa_{0} / \kappa$ sign will be at $r_{s}^{0}=5.2633$. In the range of $r_{s}>r_{s}^{0}$ the electron liquid is non-stable.

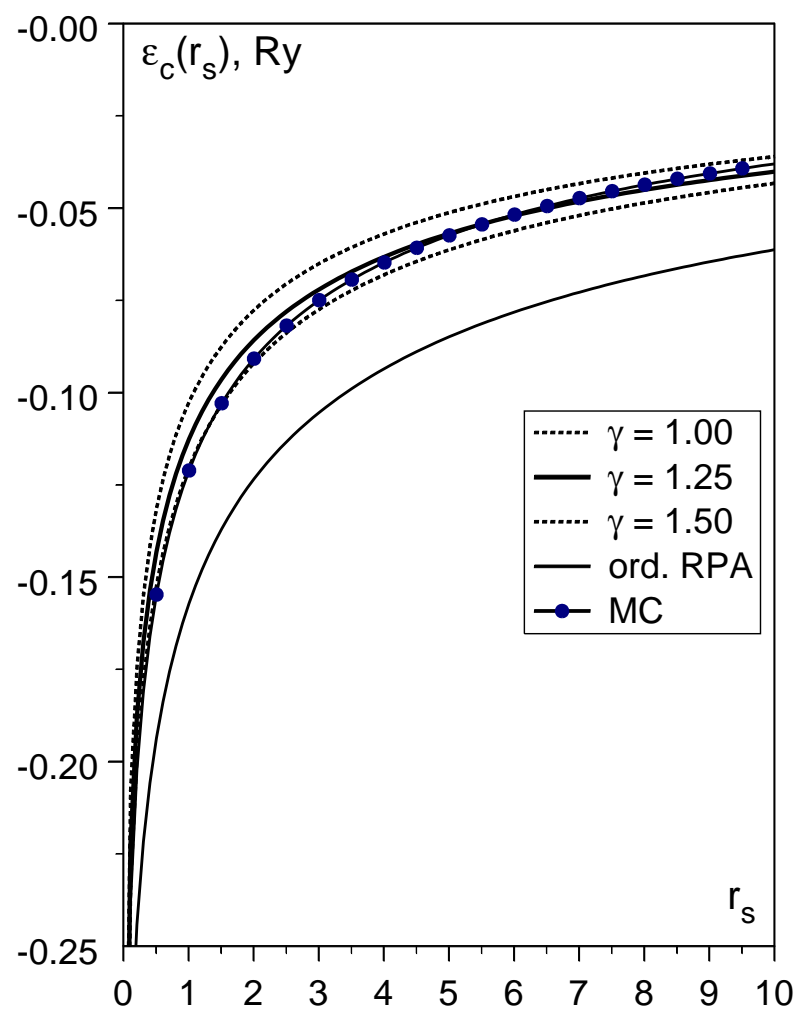

Figure 1. Correlation energy $\varepsilon_{c}\left(r_{s}\right)$ in different approaches. Model potential in variant 1 at $\gamma=1.25$ (solid curve), at $\gamma=1.0$ (top dashed curve) and at $\gamma=1.5$ (low dashed curve); Coulomb potential: MC method (black filled circles), LFCF[9] (the line with triangles).

Let us represent the calculation results of model characteristics of the electron liquid in RPA with a model potential. Figure 1 shows the dependence of correlation energy $\varepsilon_{c}\left(r_{s}\right)$ on non-ideality parameter $r_{s}$. It was calculated using the expression (19) for the first selection variant of function $\tilde{\rho}(q)$ (see table 1). Here black filled circles correspond to the MC results [5], triangles represent the best LFCF [9], low curve corresponds to the traditional RPA with Coulomb potential, top dashed curve matches up the parameter $\gamma=1.0$ and low dashed curve corresponds to $\gamma=1.5$. 
Bold solid curve is very close in the metals range to $\mathrm{MC}$ and corresponds to the case of $\gamma=1.25$. In the range of intermediate values of $r_{s}$ this curve is more close to $\mathrm{MC}$ than the calculation results with LFCF [9].

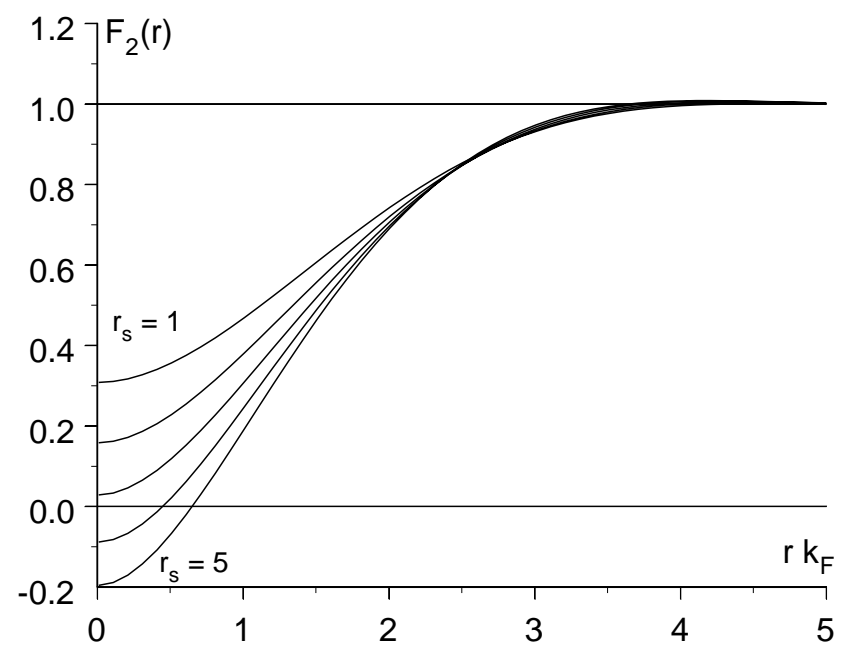

Figure 2. Binary distribution function for the model potential (variant 1) at $\gamma=1.25$.

Binary distribution function in this approach $F_{2}^{\mathrm{RPA}}(\mathbf{r})=1+\mu_{2}^{\mathrm{RPA}}(\mathbf{r})$ is shown in figure 2. These results correspond to the value of $\gamma=1.25$. As we can see, $F_{2}^{\mathrm{RPA}}(\mathbf{r})$ has small negative values at short distances, and positive values are only in the range of $0 \leqslant r_{s} \leqslant 3.3$.

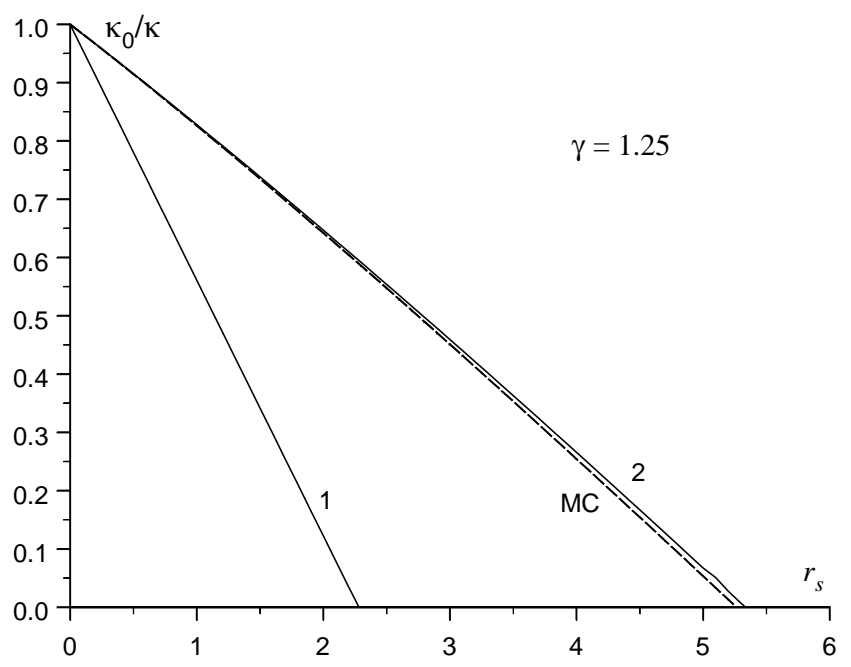

Figure 3. Inverse compressibility $\kappa_{0} / \kappa$ in different approaches. Calculated via formulae (18) (curve 1), (20) - (curve 2) and the result of MC method (dashed curve).

Figure 3 shows the dependence of inverse compressibility $\kappa_{0} / \kappa$ on $r_{s}$ in different approaches. The dashed curve corresponds to the MC method [5], other curves 
represent RPA with the model potential at $\gamma=1.25$ calculated via formulae (18) and (20).

In the represented variant the $\operatorname{LFCF} G^{0}(q)(9)$ has excessive values in the range of small wave vectors, if $1.0 \leqslant \gamma \leqslant 1.5$ (as we know, $G(q) \sim g q^{2}+\cdots$ at $q \ll 1$, where $1 / 4 \leqslant g \leqslant 1 / 3$ ). This causes invalid behavior of compressibility calculated by expression (18): at $\gamma=1.5$ the compressibility will be equivalent to zero at $r_{s}^{0} \approx 3.3$.

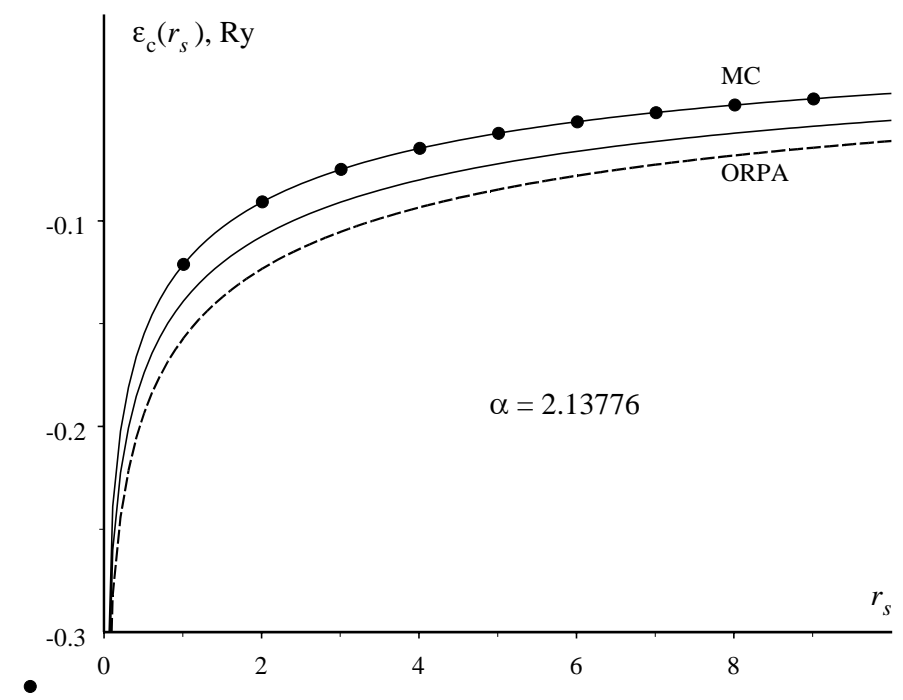

Figure 4. Correlation energy for the model potential (variant 2) at $\alpha=2.13776$ (solid curve), ordinary RPA (dashed curve), results of MC method (black filled circles).

Let us consider the same model characteristics using the other selection variant of the model potential. Namely, variant 2 , where $\tilde{\rho}(q)=\exp \left\{-q^{2} / 4 \alpha\right\}$. The dependence of the correlation energy on $r_{s}$ is shown in figure 4 . Solid bold curve corresponds to the parameter $\alpha=2.13776$. To make a comparison, the dependence of correlation energy in traditional RPA and the calculation results of MC method [5] are shown. In the range of small $r_{s}$ in this variant, $\varepsilon_{c}\left(r_{s}\right)$ is better described than in the variant 1. But in the range of large $r_{s}$ values we have the opposite situation: better results are received from variant 1 . The same situation is observed for a binary function: the results improve for a small $r_{s}$ (for an example, at $r_{s}=1$ we have $F_{2}(0)=0.276$ which corresponds to the better results obtained by other authors [10]), and become worse for large $r_{s}$. But in both selection variants of $\tilde{\rho}(q)$ all the results are certainly better than in ordinary RPA (ORPA) with Coulomb potential.

The fact that $\tilde{\rho}_{1}(q)$ better describes the model at a large value of $r_{s}$ and that $\tilde{\rho}_{2}(q)$ is better for small $r_{s}$, may be used for building a model potential as a superposition of the first and the second variants. To amend the compressibility we will use variant 1 at $\gamma=1.5$. To describe a model in a wide range of $r_{s}$ we shall build an effective potential in which

$$
\tilde{\rho}(q)=A\left(r_{s}\right) \tilde{\rho}_{2}(q)+\left[1-A\left(r_{s}\right)\right] \tilde{\rho}_{1}(q),
$$


or

$$
\tilde{\rho}^{2}(q)=A\left(r_{s}\right) \tilde{\rho}_{2}^{2}(q)+\left[1-A\left(r_{s}\right)\right] \tilde{\rho}_{1}^{2}(q),
$$

where coefficient $A\left(r_{s}\right)$ is a function of $r_{s}$ such as $A\left(r_{s}\right)=\delta *\left(1-r_{s} / 10\right), \rho_{1}(q)=$ $\gamma\left[\gamma^{2}+q^{2}\right]^{-1 / 2}, \quad \rho_{2}(q)=\exp \left(-\frac{1}{4 \alpha} q^{2}\right), \gamma=5 / 4$, and $\alpha=2.13776$

The compressibility which is calculated by the polarization operator, is defined identically in both variants:

$$
\frac{\kappa_{0}}{\kappa}=1-\frac{4 r_{s}}{\pi \eta}\left\{\frac{A\left(r_{s}\right)}{2 \alpha}+\frac{1-A\left(r_{s}\right)}{\gamma^{2}}\right\} .
$$

If $\delta=0.8$, then compressibility vanishes near $r_{s}^{0}=4.32$.

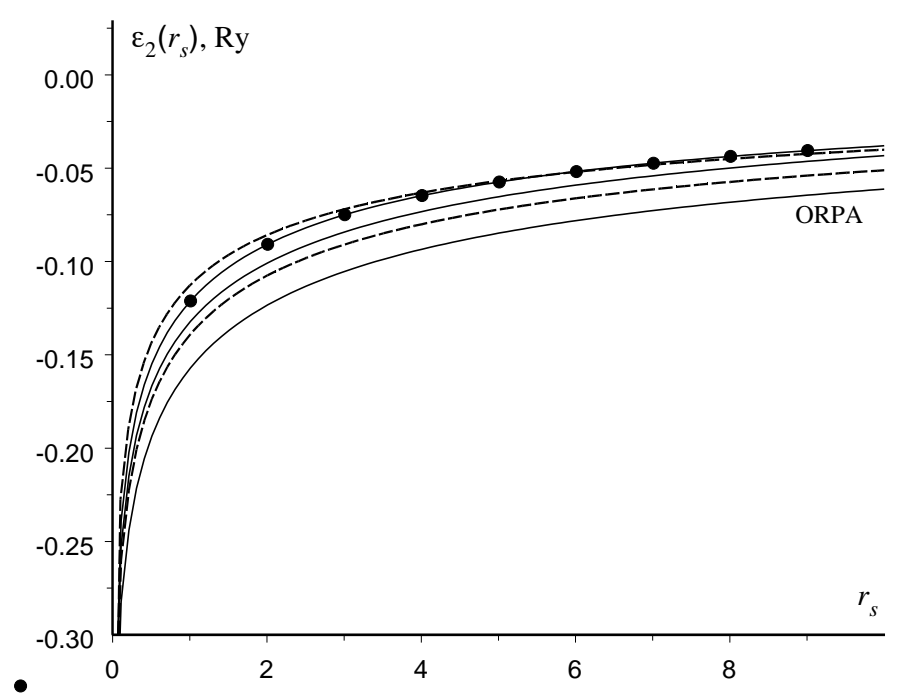

Figure 5. Correlation energy for the model potential corresponding to formula (23) (top solid curve), model potential in variant 1 at $\gamma=1.25$ (top dashed curve), model potential in variant 2 at $\alpha=2.13776$ (low dashed curve), Coulomb potential via MC method (black filled circles), ordinary RPA for Coulomb potential (low solid curve).

For all $r_{s}$, the correlation energy, calculated in RPA according to the model potential (23), better conforms to the MC method results [5] than variant 2, as shown in figure 5 . The dash curves represent the correlation energy top for variant 1 $\gamma=1.25$ and the lower curves for variant $2 \alpha=2.13776$. The binary function for this approximation has a better behavior than in variant 2 but it has a small negative value at $r=0$ for intermediate and large $r_{s}$. To amend the binary distribution function for a wide region of $r_{s}$ we must use the next approximation over RPA because this approach turns out to be deficient. The compressibility, calculated by equation (24), is closer to the compressibility obtained via MC method [5].

Let us consider another important but poorly investigated one-particle characteristic of the ELM, i.e., momentum distribution function [11,12]. We can use only the case of absolute zero temperature to investigate the effect of the short-range correlation rather than the temperature smearing of the Fermi surface. 
According to the definition

$$
n_{\mathbf{k}, s}=\left\langle a_{k, s}^{+} a_{k, s}\right\rangle_{H}=Z^{-1}(\mu) S p\left\{a_{k, s}^{+} a_{k, s} \mathrm{e}^{-\beta(\hat{H}-\mu \hat{N})}\right\}
$$

where $\mu$ is chemical potential correspondence to the given particle density and temperature, $Z(\mu)$ is the partition function. As it follows from the formula $(25), n_{\mathbf{k}, s}$ can be represented in the form of variation derivative of free energy $F$ with respect to the variable $\varepsilon_{k}=\hbar^{2} k^{2} / 2 m$,

$$
n_{\mathbf{k}, s}=\left.\frac{1}{2} \frac{\delta}{\delta \varepsilon_{k}} \Omega(\mu)\right|_{\mu}=\frac{1}{2}\left\{\frac{\delta \Omega(\mu)}{\delta \varepsilon_{k}}-\frac{\delta \mu}{\delta \varepsilon_{k}} \frac{d \Omega(\mu)}{d \mu}\right\}=\frac{1}{2} \frac{\delta F}{\delta \varepsilon_{k}},
$$

because $\delta \Omega / \delta \mu=-\mu N$. In the $T=0$ case, $F$ coincides with the average energy which is determined by formula (10). Thus, for the model potential in RPA case we obtain the representation

$$
n_{\mathbf{k}, s}=n_{\mathbf{k}, s}^{0}-\frac{1}{4 \beta V} \sum_{\mathbf{q}, \nu} V_{q}\left\{1+\frac{V_{\mathrm{ef}}}{V} \mu_{2}^{0}(x,-x)\right\}^{-1} \frac{\delta \mu_{2}^{0}(x,-x)}{\delta \varepsilon_{k}},
$$

in which $n_{\mathbf{k}, s}^{0}$ is electron momentum distribution function of an ideal system,

$$
\frac{\delta}{\delta \varepsilon_{k}} \mu_{2}^{0}(x,-x)=2 \sum_{\sigma= \pm 1} \frac{n_{\mathbf{k}, s}^{0}-n_{\mathbf{k}-\mathbf{q} \sigma, s}^{0}}{\left\{i \nu \sigma+\varepsilon_{\mathbf{k}}-\varepsilon_{\mathbf{k}-\mathbf{q} \sigma}\right\}^{2}}
$$

At the first glance the deviation $\left|n_{\mathbf{k}, s}-n_{\mathbf{k}, s}^{0}\right|$, calculated by formula (27) will exceed the similar deviation for the Coulomb potential, due to a slighter screening in (27). But the integral for $\partial \mu_{2}^{0}(x,-x) / \partial \varepsilon_{k}$ over the frequency is equal to zero. Therefore, formula (27) can be presented in an equivalent form

$$
\begin{aligned}
n_{\mathbf{k}, s}= & n_{\mathbf{k}, s}^{0}-\frac{1}{4 \beta V^{2}} \sum_{\mathbf{q}, \nu} V_{q}^{2} \tilde{\rho}^{2}(q) \hat{\mu}_{2}^{0}(x,-x) \frac{\delta \hat{\mu}_{2}^{0}(x,-x)}{\delta \varepsilon_{k}} \times \\
& \times\left\{1+\frac{V_{q}}{V} \tilde{\rho}^{2}(q) \hat{\mu}_{2}^{0}(x,-x)\right\}^{-1} .
\end{aligned}
$$

From formula (29) it follows that deviation $\left|n_{\mathbf{k}, s}-n_{\mathbf{k}, s}^{0}\right|$ will be smaller than for Coulomb potential. Going over to undimentional variables $\left(k=|\mathbf{k}| / k_{\mathrm{F}}, q, u\right)$ and integrating over angular variables of vector $\mathbf{q}$ we obtain a representation in the form of two-dimensional integrals. At $k<1$

$$
\begin{aligned}
n_{\mathbf{k}}=1-\frac{4 \pi \alpha^{2}}{k} \int_{0}^{\infty} & \left\{\int_{1-k}^{1+k} \frac{\mathrm{d} q}{q} \frac{I_{2}^{(0)}(q, u) \tilde{\rho}^{2}(q)}{q^{2}+4 \pi \alpha I_{2}^{(0)}(q, u) \tilde{\rho}^{2}(q)}\right. \\
\times & {\left[-\frac{k+q / 2}{(k+q / 2)^{2}+u^{2}}+\frac{\frac{1-k^{2}}{2 q}}{\left(\frac{1-k^{2}}{2 q}\right)^{2}+u^{2}}\right]+}
\end{aligned}
$$




$$
\begin{aligned}
& +\int_{1+k}^{\infty} \frac{\mathrm{d} q}{q} \frac{I_{2}^{(0)}(q, u) \tilde{\rho}^{2}(q)}{q^{2}+4 \pi \alpha I_{2}^{(0)}(q, u) \tilde{\rho}^{2}(q)} \\
& \left.\times\left[-\frac{k+q / 2}{(k+q / 2)^{2}+u^{2}}+\frac{q / 2-k}{(q / 2-k)^{2}+u^{2}}\right]\right\},
\end{aligned}
$$

at $k>1$

$$
n_{\mathbf{k}}=\frac{4 \pi \alpha^{2}}{k} \int_{k-1}^{k+1} \frac{\mathrm{d} q}{q} \int_{0}^{\infty} \frac{\mathrm{d} u I_{2}^{(0)}(q, u) \tilde{\rho}^{2}(q)}{q^{2}+4 \pi \alpha I_{2}^{(0)}(q, u) \tilde{\rho}^{2}(q)}\left[-\frac{k-q / 2}{(k-q / 2)^{2}+u^{2}}+\frac{\frac{k^{2}-1}{2 q}}{\left(\frac{k^{2}-1}{2 q}\right)^{2}+u^{2}}\right],
$$

where $\alpha=r_{s} \pi^{-2} \eta^{-1}$.

The results of numerical calculations are given in figure 6 , where dashed curves correspond to the Coulomb potential [11]. Therefore, the correct model consideration of short-range correlation decreases the deviation $\left|n_{\mathbf{k}, s}-n_{\mathbf{k}, s}^{0}\right|$. Thus the model approach, represented in this paper can be used as a simple and reliable method for the calculation of electron momentum distribution in a metallic region. The relative deviation of $n_{k}$ at $k \rightarrow 1-0$ for $r_{s}=9$ takes the value of $20 \%$.

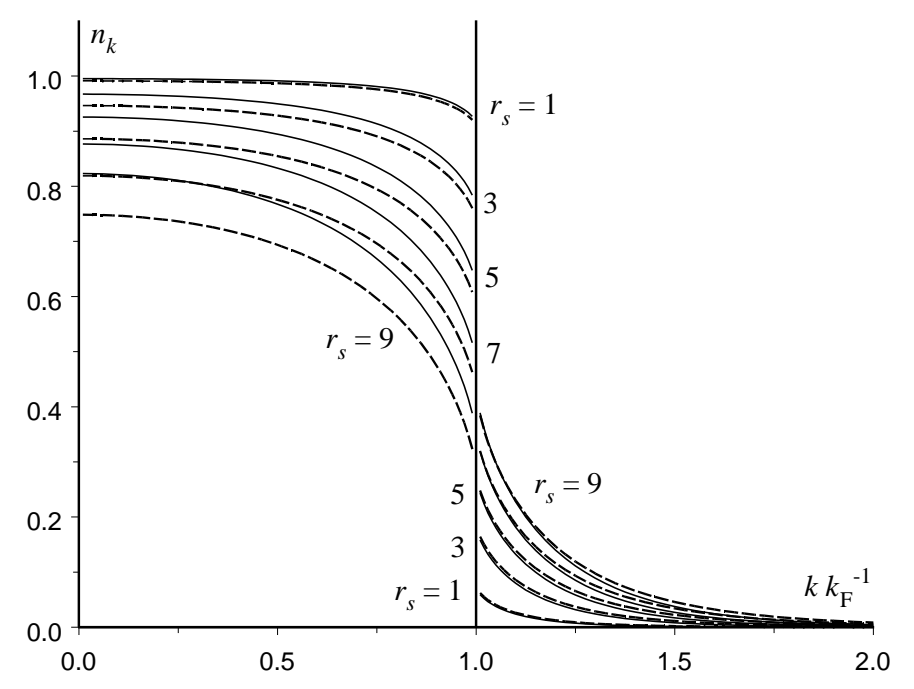

Figure 6. Momentum distribution function for the model potential (solid curves) and Coulomb potential (dashed curves) at $r_{s}=1,3,5,7,9$.

\section{Free energy of the electron liquid model}

Let us calculate the free energy of the ELM with temperatures minor in the comparison to the temperature of degeneration. To this end we will use the model potential with temperature independent parameters. As it is well known [7], the contribution of interaction to $F$ is defined by the charging energy 


$$
F-F_{0}=(2 \beta V)^{-1} \sum_{\mathbf{q}, \nu} V_{q} \int_{0}^{1} \mathrm{~d} \lambda \mu_{2}^{\lambda}(x,-x) .
$$

According to our model approach, function $\mu_{2}^{\lambda}(x,-x)$ should be calculated according to the potential $\lambda V_{\text {ef }}(\mathbf{r})$ with finite temperature.

In this paper we show the results of calculations in the form of a series by dimensionless parameter $\left(\pi T^{*}\right)^{2}$, where $T^{*} \equiv k_{\mathrm{B}} T / \varepsilon_{\mathrm{F}}$ is dimensionless temperature. Using a power series of function $\mu_{2}^{\lambda}(x,-x)$ by this parameter obtained using Zommerfeld method in the linear approximation we have

$$
F=N\left\{\varepsilon\left(r_{s}\right)-\left(\pi T^{*}\right)^{2} \varepsilon_{2}\left(r_{s}\right)\right\} \mathrm{Ry},
$$

where $\varepsilon\left(r_{s}\right)$ is the average energy in Rydberg per electron at $T=0 \mathrm{~K}$ and the correction $\varepsilon_{2}\left(r_{s}\right)$ in the RPA is equal to:

$$
\begin{aligned}
& \varepsilon_{2}\left(r_{s}\right)= \frac{\eta^{2}}{r_{s}^{2}}+\frac{\eta}{2 \pi^{2} r_{s}} \int_{0}^{\infty} \mathrm{d} u \int_{0}^{\infty} \mathrm{d} q q I_{2}^{(2)}(q, u)\left[1+\frac{4 r_{s}}{\pi \eta} \frac{I_{2}^{(0)}(q, u)}{q^{2}} \tilde{\rho}^{2}(q)\right]^{-1}, \\
& I_{2}^{(0)}(q, u)= \frac{3 N}{2 \varepsilon_{\mathrm{F}}} \frac{1}{2}\left\{1+\frac{1}{2 q}\left(1+u^{2}-\frac{q^{2}}{4}\right) \ln \frac{u^{2}+(1+q / 2)^{2}}{u^{2}+(1-q / 2)^{2}}\right. \\
&\left.-u \sum_{\sigma= \pm 1} \operatorname{arctg}\left[u^{-1}\left(1+\frac{\sigma}{2} q\right)\right]\right\}, \\
& I_{2}^{(2)}(q, u)=\frac{1}{2}\left\{\frac{1}{2 q} \ln \frac{u^{2}+(1+q / 2)^{2}}{u^{2}+(1-q / 2)^{2}}-\frac{1}{q} \sum_{\sigma= \pm 1} \sigma\left(1+\sigma \frac{q}{2}\right)\left[u^{2}+(1+\sigma q / 2)^{2}\right]^{-1}\right\} .
\end{aligned}
$$

The first term in formula (33) caused by the temperature dependence of free energy of ideal system. In the approximation (32) we obtain an expression for heat capacity of the model:

$$
C_{v}=-T \frac{\mathrm{d}^{2} F}{\mathrm{~d} T^{2}}=2 N \pi^{2} T^{*}\left(\frac{r_{s}}{\eta}\right)^{2} k_{\mathrm{B}} \varepsilon_{2}\left(r_{s}\right)+\cdots .
$$

Utilizing the well known relation between heat capacity and density of states on the Fermi surface $N_{\mathrm{F}}[7]$

$$
\frac{C_{v}}{V}=\frac{\pi^{2}}{3} k_{\mathrm{B}} T N_{\mathrm{F}}
$$

From formula (34) and (35) we obtain

$$
N_{\mathrm{F}}=4 N_{\mathrm{F}}^{\mathrm{id}}\left(\frac{r_{s}}{\eta}\right)^{2} \varepsilon_{2}\left(r_{s}\right)
$$


where $N_{\mathrm{F}}^{\mathrm{id}}=k_{\mathrm{F}}^{3}\left(2 \pi^{2} \varepsilon_{\mathrm{F}}\right)^{-1}$ is the density of states of an ideal system. Figure 7 shows the dependence of relative density of states $N_{\mathrm{F}} / N_{\mathrm{F}}^{\mathrm{id}}$ on the coupling parameter in RPA with the model potential (23) (curve 1) and with Coulomb potential (curve 2).

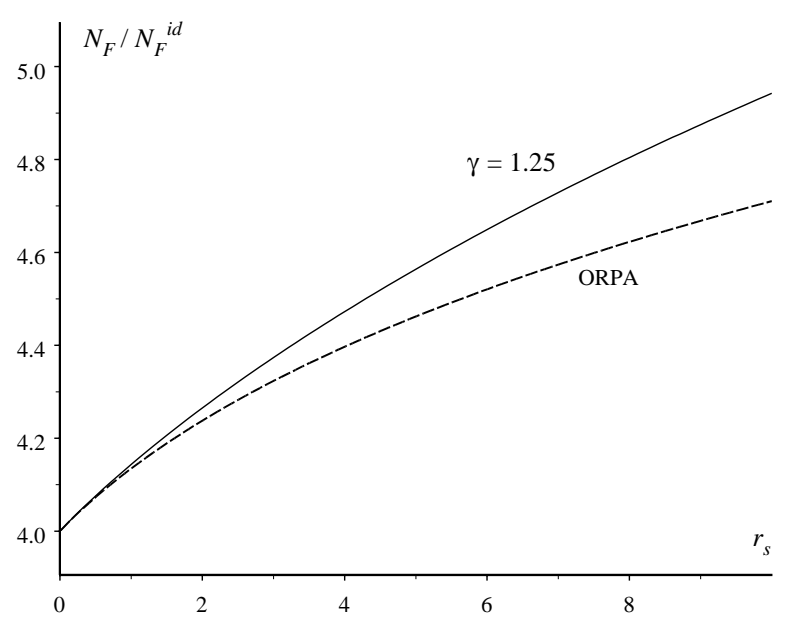

Figure 7. Dependence of relative density of states $N_{F} / N_{F}^{\mathrm{id}}$ on coupling parameter in RPA with model potential (23) (solid curve) and with Coulomb potential (dashed curve).

\section{Local-field approximation with an effective potential}

We want to achieve the correctness of all characteristics of ELM in the region of weak and intermediate non-ideality. Thus, we will overstep the RPA with model potentials. As in the case of formula (8) of the traditional perturbation theory, by using the summing of diagrams in the reference system approach [8] for the function $\mu_{2}(x,-x)$ we obtain a representation

$$
\mu_{2}(x,-x)=\mu_{2}^{0}(x,-x)\left\{1+\frac{V_{\text {ef }}(q)}{V} \mu_{2}^{0}(x,-x)\left[1-G_{\mathrm{M}}(x)\right]\right\}^{-1} .
$$

Here $G_{\mathrm{M}}(x)$ is a LFCF for the system of particles with a model potential $V_{\mathrm{ef}}(q)$. Equating expressions (8) and (37) we obtain a relation

$$
G(x)=1-\tilde{\rho}^{2}(\mathbf{q})\left\{1-G_{\mathrm{M}}(x)\right\}
$$

which may be considered as one of the simple ways of calculating the LFCF. We use the Geldart-Taylor approximation (these authors for the first time have calculated the static correction for polarization operator for Coulomb potential in the linear approximation [13]) because $V_{\mathrm{ef}}(q)$ is a weak potential. In this approximation we obtain

$$
G_{\mathrm{M}}^{(1)}(x)=-\left\{2 \beta V_{\mathrm{ef}}(q)\right\}^{-1}\left\{\mu_{2}^{0}(x,-x)\right\}^{-2} \sum_{x_{1}} V_{\mathrm{ef}}\left(q_{1}\right) \mu_{4}^{0}\left(x,-x, x_{1},-x_{1}\right),
$$


where $\mu_{4}^{0}\left(x,-x, x_{1},-x_{1}\right)$ is a four-particle semiinvariant correlation function of the reference system [8]. Performing the summing over frequencies we get the following representation:

$$
\begin{aligned}
G_{\mathrm{M}}^{(1)}(x)= & \left\{V_{\mathrm{ef}}(q)\right\}^{-1}\left\{\mu_{2}^{0}(x,-x)\right\}^{-2} \operatorname{Re} \sum_{\mathbf{k}_{1}, \mathbf{k}_{2} ; s} V_{\mathrm{ef}}\left(\mathbf{k}_{1}-\mathbf{k}_{2}\right) \\
& \times\left\{n_{\mathbf{k}_{1}, s}^{0}-n_{\mathbf{k}_{1}-\mathbf{q}, s}^{0}\right\}\left\{n_{\mathbf{k}_{2}, s}^{0}-n_{\mathbf{k}_{2}-\mathbf{q}, s}^{0}\right\}\left[\mathrm{i} \nu+\varepsilon_{\mathbf{k}_{1}}-\varepsilon_{\mathbf{k}-\mathbf{q}_{1}}\right]^{-1} \\
& \times\left\{\left[\mathrm{i} \nu+\varepsilon_{\mathbf{k}_{1}}-\varepsilon_{\mathbf{k}_{1}-\mathbf{q}}\right]^{-1}-\left[\mathrm{i} \nu+\varepsilon_{\mathbf{k}_{2}}-\varepsilon_{\mathbf{k}_{2}-\mathbf{q}}\right]^{-1}\right\}
\end{aligned}
$$

Let us investigate the asymptote of (40) for small and large values of wave vectors. In a long-wave limit we substitute $\tilde{\rho}(q)=1$ and use the series $n_{\mathbf{k}-\mathbf{q}, s}^{0}=$ $n_{\mathbf{k}, s}^{0}+\mathrm{d} n_{\mathbf{k}, s}^{0} / \mathrm{d} \varepsilon_{k}\left(\varepsilon_{\mathbf{k}-\mathbf{q}}-\varepsilon_{\mathbf{k}}\right)+\cdots$ Going over from the sums over vectors $\mathbf{k}_{1}, \mathbf{k}_{2}$ to integrals in a spherical system of coordinates we reduce the calculation to a onedimensional integral

$$
G_{\mathrm{M}}^{(1)}(x)=g_{1}(\nu)\left(\frac{q}{k_{\mathrm{F}}}\right)^{2}+\cdots, q \ll k_{\mathrm{F}} .
$$

In a static limit we have

$$
g_{1}(0)=\frac{1}{8} \int_{-1}^{1} \mathrm{~d} t \tilde{\rho}^{2}\left([2(1-t)]^{1 / 2}\right)=\frac{1}{8} \int_{0}^{2} \mathrm{~d} q q \tilde{\rho}^{2}(q),
$$

and in the large frequencies limit we have

$$
g_{1}(\infty)=\frac{3}{40} \int_{0}^{2} \mathrm{~d} q q\left(q^{2}-1\right) \tilde{\rho}^{2}(q) .
$$

Here $t=\cos \theta$ cosine of the angle between vectors $\mathbf{k}_{1}$ and $\mathbf{k}_{2}$. At $\tilde{\rho}(q)=1$ we obtain the corresponding asymptote for LFCF in Geldart-Taylor approximation for the system with Coulomb potential $(g(0)=1 / 4, g(\infty)=3 / 20)$. In the large wave vector limit we obtain the following asymptote:

$$
\begin{aligned}
G_{\mathrm{M}}^{(1)}(x)= & \frac{1}{2}-N^{-2} q^{-4} V_{\mathrm{ef}}^{-1}(q) \sum_{\mathbf{k}_{1}, \mathbf{k}_{2} ; s} V_{\mathrm{ef}}\left(\mathbf{k}_{1}-\mathbf{k}_{2}\right) \\
& \times n_{\mathbf{k}_{1}, s}^{0} n_{\mathbf{k}_{2}, s}^{0}\left\{\left(\mathbf{k}_{1} \mathbf{q}\right)^{2}+\left(\mathbf{k}_{2} \mathbf{q}\right)^{2}-2\left(\mathbf{k}_{1} \mathbf{q}\right)\left(\mathbf{k}_{2} \mathbf{q}\right)\right\}+\cdots \\
= & \frac{1}{2}-\frac{3}{4} \tilde{\rho}^{-2}(q) \int_{0}^{1} \mathrm{~d} k_{1} k_{1}^{2} \int_{0}^{1} \mathrm{~d} k_{2} k_{2}^{2} \int_{-1}^{1} \mathrm{~d} t \tilde{\rho}^{2}\left(\left[k_{1}^{2}+k_{2}^{2}-2 k_{1} k_{2} t\right]^{1 / 2}\right)+\cdots
\end{aligned}
$$

According to the last formula for Coulomb potential $\tilde{\rho}(q)=1$ the LFCF asymptote is equal to $1 / 3$ [4]. For the potentials of quantum packet type, $G_{\mathrm{M}}^{(1)}(x)$ acquires 
negative values for the large wave vectors and essentially differs from LFCF in the weakly coupled electron liquid case. However, according to formula (39) in the LFCF for ELM $G(x)$ in such an approximation remains positive and tends an asymptote

$$
G^{(1)}(x) \Rightarrow 1-\frac{1}{2} \tilde{\rho}^{2}(q)-\frac{3}{4} \int_{0}^{1} \mathrm{~d} k_{1} k_{1}^{2} \int_{0}^{1} \mathrm{~d} k_{2} k_{2}^{2} \int_{-1}^{1} \mathrm{~d} t \tilde{\rho}^{2}\left(\left[k_{1}^{2}+k_{2}^{2}-2 k_{1} k_{2} t\right]^{1 / 2}\right)
$$

at $q \gg k_{\mathrm{F}}$ (at $\tilde{\rho}(q)=1$ this limit is equal to $\left.1 / 3\right)$. The numerical calculation of $G_{\mathrm{M}}^{(1)}(x)$ using (40) can be done for an arbitrary effective potential. In the $T=0 \mathrm{~K}$ case and at any given $\tilde{\rho}(q)$, the calculation reduces to a five-dimensional integral. Let us proceed from sums to integrals and use the dimensionless variables. We use the cylindric coordinate system (axis OZ is parallel to vector $\mathbf{q}$ ). Since $\mathbf{k}_{i}=\left(k_{\mathrm{F}} \rho_{i}, k_{\mathrm{F}} z_{i}, \varphi_{i}\right)$, then $k_{\mathrm{F}}^{-2}\left(\mathbf{k}_{i}, \mathbf{q}\right)=z_{i} q, k_{\mathrm{F}}^{-2}\left(\mathbf{k}_{1}-\mathbf{k}_{2}\right)^{2}=\rho_{1}^{2}+\rho_{2}^{2}+\left(z_{1}-z_{2}\right)^{2}-2 \rho_{1} \rho_{2} \cos \left(\varphi_{1}-\varphi_{2}\right)$. With substitution $\varphi=\varphi_{1}-\varphi_{2}$ after integration over $\varphi_{2}$ we transform the expression into the form

$$
\begin{aligned}
G_{\mathrm{M}}^{(1)}(x)= & \tilde{\rho}^{-2}(q) I_{2,0}^{-2}(q, u)(16 \pi)^{-1} \\
& \times \iint_{-1}^{1} \mathrm{~d} z_{1} \mathrm{~d} z_{2} \int_{0}^{\left(1-z_{1}^{2}\right)^{1 / 2}} \mathrm{~d} \rho_{1} \rho_{1} \int_{0}^{\left(1-z_{2}^{2}\right)^{1 / 2}} \mathrm{~d} \rho_{2} \rho_{2} \int_{0}^{2 \pi} \mathrm{d} \varphi \sum_{\sigma_{1}, \sigma_{2}= \pm 1} \sigma_{1} \sigma_{2} \\
& \times \tilde{\rho}^{2}\left(P_{\sigma_{1} \sigma_{2}}^{1 / 2}\right) P_{\sigma_{1} \sigma_{2}}^{-1}\left\{z_{1}^{\sigma}-z_{2}^{\sigma}\right\}\left\{\left(z_{1}^{\sigma}\right)^{2} z_{2}^{\sigma}-u^{2}\left[2 z_{1}^{\sigma}+z_{2}^{\sigma}\right]\right\} \\
& \times\left\{u^{2}+\left(z_{1}^{\sigma}\right)^{2}\right\}^{-2}\left\{u^{2}+\left(z_{2}^{\sigma}\right)^{2}\right\}^{-1},
\end{aligned}
$$

where $z_{1}^{\sigma} \equiv z_{1}+1 / 2 \sigma_{1} q, z_{2}^{\sigma} \equiv z_{2}+1 / 2 \sigma_{2} q, P_{\sigma_{1} \sigma_{2}} \equiv \rho_{1}^{2}+\rho_{2}^{2}+\left[z_{1}^{\sigma}-z_{2}^{\sigma}\right]^{2}-2 \rho_{1} \rho_{2} \cos \varphi$.

In the case of variants 1,3 and 4 (see table 1 ) the calculations can be reduced to a two-dimensional integral, since the integration over variables $\varphi_{i}$ and $\rho_{i}$ can be performed in an analytical form. To illustrate we show an expression for $G_{\mathrm{M}}^{(1)}(x)$ for variant 1 in which $\tilde{\rho}^{2}(q)=\gamma^{2}\left(\gamma^{2}+q^{2}\right)^{-1}$

$$
\begin{aligned}
G_{\mathrm{M}}^{(1)}(q, u)= & \left(\gamma^{2}+q^{2}\right)\left[2 \gamma I_{2,0}(q, u)\right]^{-2} \iint_{-1}^{1} \mathrm{~d} z_{1} \mathrm{~d} z_{2} \sum_{\sigma_{1}, \sigma_{2}= \pm 1} \sigma_{1} \sigma_{2} \\
& \times \Psi\left(z_{1}+\frac{\sigma_{1}}{2} q ; z_{2}+\frac{\sigma_{2}}{2} q ; 1-z_{1}^{2} ; 1-z_{2}^{2}\right) .
\end{aligned}
$$

Here the following notations were used:

$$
\begin{aligned}
\left.\Psi_{(} a ; b ; w ; v\right) & =\Phi_{0}(a ; b ; w ; v)-\Phi_{\gamma}(a ; b ; w ; v) \\
\Phi_{\gamma}(a ; b ; w ; v) & =\frac{1}{8}(a-b) J\left(s_{\gamma}^{2}, w, v\right)\left(b^{2}+u^{2}\right)^{-1}\left(a^{2}+u^{2}\right)^{-2}\left\{a^{2} b-u^{2}(2 a+b)\right\} \\
J\left(s_{\gamma}^{2}, w, v\right) & =\frac{1}{2}\left[W\left(s_{\gamma}^{2}, w, v\right)-w-v-s_{\gamma}^{2}\right]
\end{aligned}
$$




$$
\begin{aligned}
+ & w \ln \left\{\frac{1}{2} s_{\gamma}^{-2}\left[W\left(s_{\gamma}^{2}, w, v\right)+s_{\gamma}^{2}+v-w\right]\right\} \\
& +v \ln \left\{\frac{1}{2} s_{\gamma}^{-2}\left[W\left(s_{\gamma}^{2}, w, v\right)+s_{\gamma}^{2}+w-v\right]\right\} \\
W\left(s_{\gamma}^{2}, w, v\right)= & \left\{s_{\gamma}^{4}+2 s_{\gamma}^{2}(w+v)+(w-v)^{2}\right\}^{1 / 2},
\end{aligned}
$$

where $s_{\gamma}^{2}=\gamma^{2}+(a-b)^{2}$ and $\Phi_{0}(a ; b ; w ; v)$ coincide with $\Phi_{\gamma}(a ; b ; w ; v)$ at $\gamma=0$.

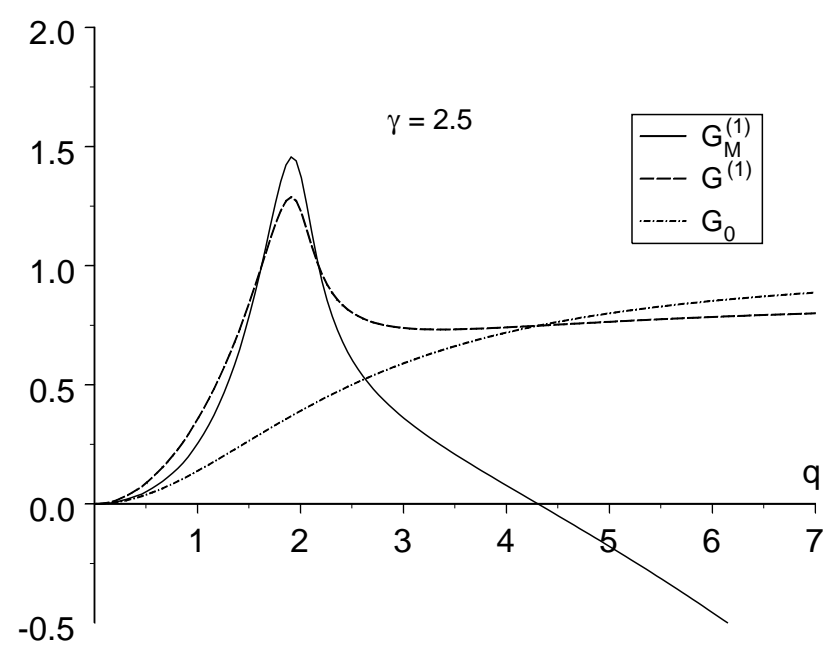

Figure 8. Local-field correction functions $G_{M}^{(1)}(x)$ and $G^{(1)}(x)$ in the static case for the system with model potential.

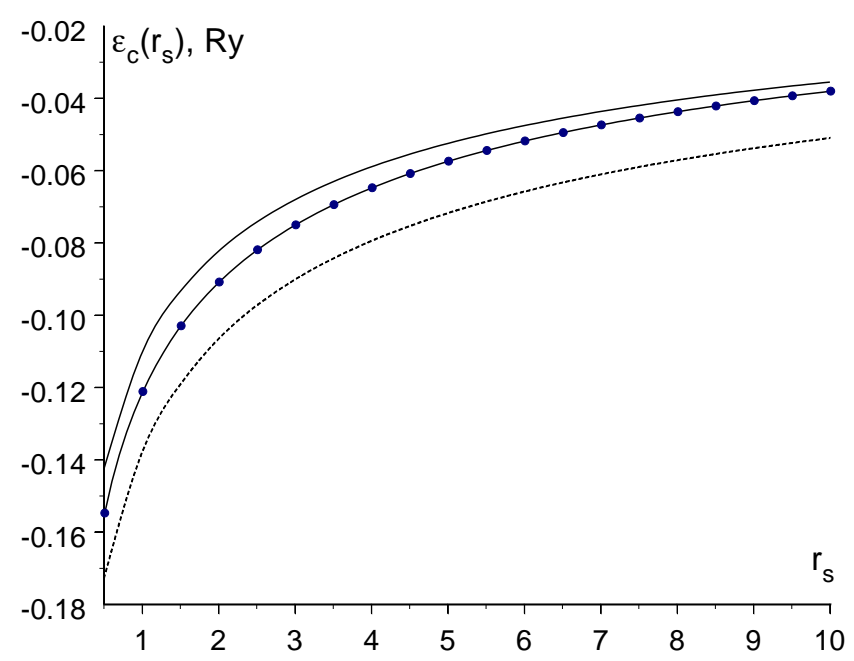

Figure 9. Correlation energy calculated via LFCF for the model potential (top solid curve), Coulomb potential: ordinary RPA (low curve), MC method (the line with filled circles).

Figure 8 shows the properties of functions $G_{\mathrm{M}}^{(1)}(x)$ and $G^{(1)}(x)$ in the static case. For $\gamma=2.5$ function, $G^{(1)}(x)$ have a long-wave asymptote $g\left(q / k_{\mathrm{F}}\right)^{2}$, where 


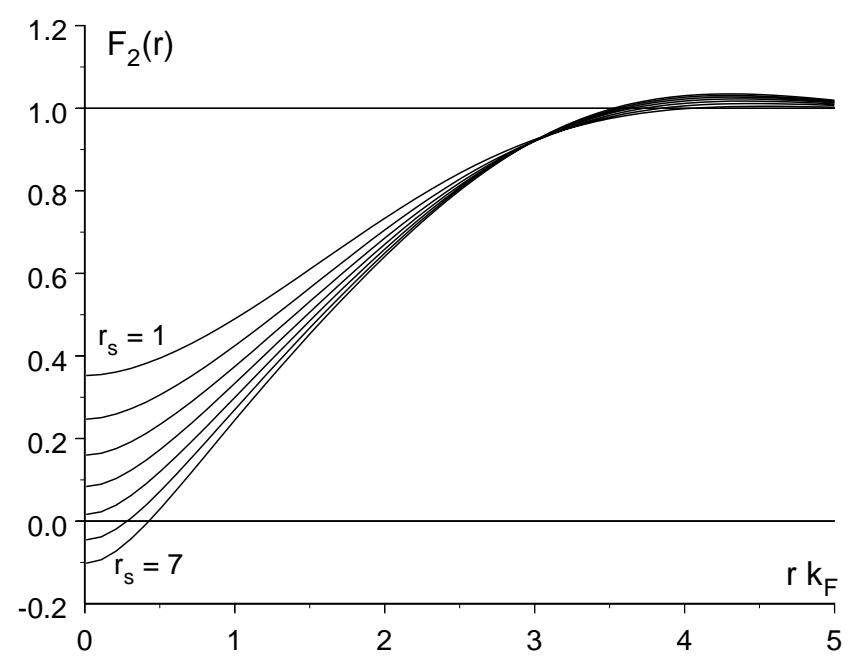

Figure 10. Binary distribution function calculated via LFCF for the model potential.

$g \approx 1 / 3$, which gives $r_{s}^{0} \approx 5.0$ and provides a correct behaviour of compressibility. The dependence of the correlation energy in this approximation is shown in figure 9 : the curve with filled circles corresponds to the results obtained with MC method [5], dashed line corresponds to RPA with the model potential, solid curve corresponds to $\operatorname{LFCF} G_{\mathrm{M}}^{(1)}(x)$ and to the model potential (23). The average deviation from the results obtained with $\mathrm{MC}$ method in the range of $0 \leqslant r_{s} \leqslant 10$ is $8 \%$, while the average deviation in RPA with the model potential is equal to $26 \%$. The binary distribution function in this approximation has a correct behaviour in the range of $0 \leqslant r_{s} \leqslant 5.5$ (figure 10 ).

\section{Conclusions}

The modern theory of strong degenerated systems is based on the local-field concept. However, looking for alternative methods remains urgent because the microscopic local-field correction function theory is still unfinalized. The model approach, based on regularization of Coulomb potential was proposed by the authors earlier. In this paper we suggest the criterions of selecting the form of the model potential, which correct describes the set of characteristics of the electron liquid model (i.e., the correlation energy, the binary distribution function, the compressibility) in a wide region Wigner's parameter $r_{s}$. We have analyzed several weak long-range interaction potentials between particles, which have an asymptote $e^{2} / r$ at large distances and is regular when $r \rightarrow 0$. The application of these potentials gives good results for a small, intermediate and strong non-ideality of the system by using the methods typical of weakly non-ideal systems. As it follows from our calculations, one of the best model potentials are possessed by the Fourier-transform $V_{\text {ef }}(q)=4 \pi e^{2} q^{-2} \exp \left\{-(2 \alpha)^{-1}\left(q / k_{\mathrm{F}}\right)^{2}\right\}$ at $\alpha=2.1377 \ldots$ When utilizing the model potentials we reduce to the calculation of the characteristics like the momentum distribution of electron and local-field correction function. On the whole, the regu- 
larization of the Coulomb potential is a simple and promising method of calculating the characteristics of metallic systems.

\title{
References
}

1. Vavrukh M., Koval' S., Tyshko N. // Journal of Physical Studies (Lviv), 2000, vol. 4, p. 403 (in Ukrainian).

2. Ziesche P., Lehmann G. Ergebnisse in der Elektronentheorie der Metalle. Berlin, Akademie-Verlag, 1983.

3. Abramovwitz M., Stegun I. (Ed.). Handbook of Mathematical Functions with Formulas, Graphs and Mathematical Tables. USA, National bureau of standards, 1964.

4. Vavrukh M. // Fizika Nizkikh Temperatur (Kharkiv), 1966, vol. 22, p. 1005 (in Russian); Vavrukh M. // Low. Temp. Phys., 1996, vol. 22, p. 767.

5. Ceperley D.M., Alder B.J. // Phys. Rev. Lett., 1980, vol. 45, p. 566.

6. Vosko S.H., Wilk L., Nusair N. // Canad. J. Phys., 1980, vol. 58, p. 1200.

7. Pines D. Elementary Excitations in Solids (W.A.Benjamin). New York, 1964.

8. Vavrukh M., Krokhmalskii T. // Phys. stat. sol. (b), 1991, vol. 168, p. 519;

Vavrukh M., Krokhmalskii T. // Phys. stat. sol. (b), 1992, vol. 169, p. 451.

9. Ichimaru S., Utsumi K. // Phys. Rev. B, 1981, vol. 24, p. 7385.

10. Lannto L.J. // Phys. Rev. B, 1980, vol. 22, p. 1380.

11. Daniel E., Vosko S.M. // Phys. Rev., 1960, vol. 120, p. 2041.

12. Vavrukh M., Vavrukh N. // Phys. Stat. Sol. (b), 1994, vol. 186, p. 159.

13. Geldart D.I.M., Taylor R. // Canad. J. Phys., 1970, vol. 48, p. 155, 167.

\section{Регуляризація потенціалу як спосіб врахування короткосяжних кореляцій у теорії електронної рідини}

\author{
М.В.Ваврух, Н.Л.Тишко \\ Львівський національний університет імені Івана Франка, кафедра \\ астрофізики, вул. Кирила і Мефодія, 8, Львів, 79005, Україна \\ Отримано 1 квітня 2004 р. \\ В попередній роботі авторів [1] запропоновано ідею регуляризації \\ потенціалу Кулона як один із способів врахування короткосяжних ко- \\ реляцій в моделі електронної рідини. В даній роботі сформульовано \\ критерії оптимального вибору регуляризації і розраховано енерге- \\ тичні, структурні та діелектричні характеристики моделі.
}

Ключові слова: модель електронної рідини, короткосяжні кореляції, поправка на локальне поле, кореляційна енергія, бінарна функція розподілу, стисливість

PACS: 05.30.Fk 